\title{
EFECTO COMPARATIVO DE LA VIRULENCIA DE LOS HONGOS APHANOCLADIUM ALBUM (Preuss) Gams Y TOLYPOCLADIUM CYLINDROSPORUM Gams (DEUTEROMYCOTINA- HYPHOMYCETES), CONTRA LARVAS DE MOSQUITO (DIPTERA,CULICIDAE).*
}

\author{
Claudia Cristina Lopez Lastra A \\ Juan José García G., Guillermo Raúl Reboredo O
}

CEPAVE (Centro de estudios Parasitológicos y de Vectores,UNLP)..

Calle 2 \# 584-1900 La Plata Argentina.

Palabras Clave: Virulencia, Aphanocladium album, Tolypocladium cylindrosporum, larvas de Culex pipiens

Keys word: Virulence, Aphanocladium album, Tolypocladium cylindrosporum, Culex pipiens larvae

\section{RESUMEN}

El presente trabajo ha sido realizado con el objeto de evaluar el efecto potencial patogénico de conidios y blastosporas de Aphanocladiumalbumy Tolypocladium cylindrosporum contra larvas de Culex pipiens $L$.

Un alto porcentaje de mortalidad larval se observó frente a las 2 especies fúngicas, siendo para A.album, $85,6 \%$ y para T.cylindrosporum $80 \%$, con dosis de $1 x$ $10^{6}$ conidios $/ \mathrm{ml}$.

En A. album la actividad de blastosporos y conidios fué similar, mientras que para T. cylindrosporum fueron más virulentos los conidios que las blastosporas.

De los resultados obtenidos se deduce que ambas especies fúngicas tienen una elevada virulencia contra larvas de C. pipiens y sus potencialidades patogénicas justifican nuevos estudios sobre este tema.

\section{SUMMARY}

[Comparative study of Virulence of Fungi: Aphanocladium album (Preuss) Gams and Tolypocladium cylindrosporum Gams (Deuteromycotina: Hyphomycetes) Against mosquitoes larvae (Diptera: Culicidae].

The present work evaluates the pathogenic potential effect of conidia and blastospores in fungi Aphanocladium album and Tolypocladium cylindrosporum against Culex pipiens, larvae. A high larval percentage of mortality was observed in both fungi species. At a dosage of $1 \times 10^{6}$ conidialml, was $85.6 \%$ for A. album, and $80 \%$ for T.cylindrosporum.

A similar acivity was observed between conidia and blastospores for A. album while for T. cylindrosporum it was more virulent conidia than for blastospores.

Finally results revealed that both fungi species have a high virulence against $C$. pipiens larvae, so that their pathogenic potential would justify new studies on this subject.

ces clavisporus, Metarhizium anisopliae, Tolypocladium cylindrosportum y Lagenidium giganteum (Riba et al. 1986).

Tolypocladium cylindrosporum fue citado como patógeno de insectos, particularmente de larvas de

agentes potenciales para el control de mosquitos, entre ellas se pueden citar a Coelomomyces spp, Culicinomy.

Trabajo subsidiado por UNDP/WORLD BANK/WHO. Special programme for the research and training in tropical diseases. ID 890076 
mosquitos por Soares et al. (1979) y Weisser \& Pillai (1981). Su patogenicidad fue comprobada para varias especies de culicidos por Pinnock et al. (1973),Soares et al. (1979), Soares etal. (1985), Weiser y Pillai (1981), Yu et al. (1980) y Goettel (1987 a y b.).

A pesar de que han sido realizados pocos estudios referentes a su aplicacion en el campo (Gardner et al.(1986) y Pillai, 1987, Goetel 1987), los ensayos de laboratorio revelan que esta especie puede ser un potencial agente de control de mosquitos (Soares, 1982, Weisser y Pillai, 1981, Riba et al., 1986, Gottel, 1987).

Respecto de Aphanocladium album, no ha sido evaluado hasta el presente como agente patógeno de mosquitos, sólo se ha citado como patógeno de adultos de Aedes albifasciatus en Argentina (López Lastra, 1990) y existen estudios preliminares (aún no publicados) a fin de comprobar la viabilidad de esta especiey T.cylindrosporum, bajo condiciones variables de temperatura y salinidad.

El presente trabajo ha sido realizado con el objeto de evaluar el efecto patógenico de A. album, comparándolo en su virulencia con un aislamiento de T. cylindrosporum (obtenido de suelo de la Antártida), el cual ha sido evaluado en pruebas de patogenicidad preliminares contra larvas de Culex pipiens, en condiciones controladas de laboratorio, a fin de considerar la perspectiva de su posterior aplicación y evaluación en pruebas de campo.

\section{MATERIAL Y METODOS}

Para la realización de los bioensayos se procedió de igual forma con conidios y blastosporas de Tolypocladium cylindrosporzum, aislado de suelo de Antártida (NT.c.3.4. Colección Micológica CEPAVE) y con Aphanocladium aibum aislado a partir de adultos de Aedes albifasciatus (N.A.a.1.7. Col. Mic. CEPAVE).

Para cada prueba se emplearon lotes de 10 larvas de segunda estadía de Culex pipiens, procedentes de la colonia instalada en el CEPAVE (Centro de Estudios Parasitológicos y de Vectores), las que fueron colocadas en recipientes de plástico de $3 \mathrm{~cm}$. de diámetro por $8 \mathrm{~cm}$. de altura, conteniendo $10 \mathrm{ml}$. de agua destilada. Las larvas fueron alimentadas con alimento para peces (previo molido ultrafino). Cada lote fué tratado con diferentes concentraciones de conidios y blastosporas. Las dosis empleadas fueron $1 \times 10^{3}, 1 \times 10^{4}, 1 \times 10^{5}, 1 \times$ $10^{6}, 1 \times 10^{7}$, y $1 \times 10^{8}$ esporas por $\mathrm{ml}$.

Cada ensayo contó con un testigo, al que se agrego agua destilada estéril pura, realizándose 5 repeticiones de cada ensayo. Todos los recipientes con las larvas se mantuvieron a $25^{\circ} \mathrm{C}$ y $80 \%$ de H.R. El control de la mortalidad larval fué efectuado cada 24 horas durante
16 días, extrayéndose las larvas muertas para comprobar su causa. Las infecciones fúngicas se detectaron bajo microscopio óptico con contraste de fase.

Los conidios de ambas especies fúngicas se obtuvieron a partir de cultivos realizados en cápsulas de Petri con $10 \mathrm{ml}$. de medio Sabouraud dextrosa agar, con $2 \%$ de extracto de levadura (SDA-Y), incubados a $25^{\circ} \mathrm{C}$ durante 10 días. Los conidios fueron extraídos de la superficie del cultivo con un pincel de pelo de marta estéril, preparándose con ellos una suspension en agua destilada estéril, la que fué homogeneizada por agitación manual.

Las blastosporas de ambas especies se cultivaron en medio líquido Sabouraud Dextrosa caldo con $2 \%$ de extracto delevadura (SDA-Y) en Erlenmeyers, los que se mantuvieron en agitación permanente a 78 r.p.m. durante 7 días a $25^{\circ} \mathrm{C}$. El número de esporas (conidios y blastosporas) se cuantifico con una cámara de Neubauer.

Se obtuvieron datos de mortalidad larval acumulada y la mortalidad final fué corregida por la formula Abbott (1925), el tiempo letal 50 (TL 50) se obtuvo método de Biever y Hostetter (1971) y la dosis letal 50 (DL50) por el método deReed y Muench(1938).

\section{RESULTADOS}

Los datos obtenidos sobre la mortalidad de larvas, dosis letai 50 (DL 50) y el tiempo letal (TL 50)se expresan en tablas 1 y 2.

Los valores de DL 50 obtenidos con blastosporas y conidios de $A$. album fueron $1 \times 10^{3}$ blestosporas $/ \mathrm{ml}$ y $1 \times 10^{3}$ conidios/ml respectivamente. El menor TL 50 para la misma especie fué 2.25 días a una dosis y $1 \times 10^{7}$ blas./ml y 2.97 días con $1 \times 10^{8} \mathrm{con}$. $/ \mathrm{ml}$. Los mayores TL 50 obtenidos con blastosporas y conidios de $\mathrm{A}$. album fueron de 11 y 12.6 días respectivamente, con dosis de $1 \times 10^{4} \mathrm{blas} . / \mathrm{ml}$ y $1 \times 10^{3} \mathrm{con}$. $\mathrm{ml}$. Se obtuvieron porcentajes de mortalidad de $100 \%$ en larvas expuestas a dósis de $1 \times 10^{7}$ blas./ml y $1 \times 10^{5} \mathrm{con} . / \mathrm{ml}$ de $A$. album durante 5 y 14 cías respectivamente.

Los resuitados de los bioensayos realizados con blastosporas y conidios de $T$. cylindrosporum, fueron : DL 50: $5 \times 10^{4}$ blas. $/ \mathrm{ml}$ y $1 \times 10^{4} \mathrm{con} . / \mathrm{ml}$. respectivamente, siendo el menor TL 50 para blastosporas de 2.87 d!́as con la dosis: $1 \times 10^{6}$ blas./ml y el mayor TL 50 de 15 días con $1 \times 10^{3}$ blas. $/ \mathrm{ml}$. El menor TL 50 obtenicio solamente con conidios de $T$. cylindrosporum fue de 3.26 días a una dosis de $1 \times 10^{8} \mathrm{con} / \mathrm{ml}$, siendo el mayor TL 50 de 13 días con una dosis de $1 \times 10^{4} \mathrm{con} /$ $\mathrm{ml}$.

Se obtuvieron porcentajes de mortalidad de $100 \%$ con blastosporas de $T$. cylindrosporum, luego de 11 días 
de exposición de las larvas a una dosis de $1 \times 10^{8}$ blas./ml,mientras que los porcentajes no superaron el 95\% cuando se utilizo la misma dosis de conidios durante los 16 días que duró el ensayo.

\section{DISCUSION Y CONCLUSIONES}

Comparando la actividad de ambas especies fúngicas se observó un desarrollo más rápido de la infección con A. album que con T. cylindrosporum y en ambas especies fue mayor para blastosporas que para conidios presentando los dos hongos valores de DL 50 bajos, entre $1 \times 10^{3}$ y 1 x $10^{4}$ esporas/ml.

Riba et al. (1986), evaluaron la patogenicidad de un aislamiento de T. cylindrosporum contra larvas de segundo estadía de Aedes aegypti, determinando la mayor actividad de las blastosporas que de los conidios a una dosis: $1 \times 10^{7}$ esporas $/ \mathrm{ml}$. para ambos casos, mientras que hallaron un TL 50 de 4.1 días para blastosporas contra 26.6 días para los conidios.

Gardner y Pillai (1987) determinaron las potencialidades de un aislamiento de $T$. cylindrosporum a partir de Aedes austrolis de Nueva Zelandia contra larvas de esa especie. Los autores demostraron el desarrollo más rápido de las infecciones con blastosporas (TL 50:3.8 días) que con conidios (TL 50: 7.9 días), utilizando una dosis: $2 \times 10^{5}$ esporas/ml. La DL 50 determinada para las blastosporas de este aislamiento varía entre $9.5 \times 10^{4}$ y $4.2 \times 10^{5} \mathrm{blas} . / \mathrm{ml}$, mientras que con los conidios correspondió a $3 \times 10^{6} \mathrm{con} / \mathrm{ml}$, , siendo menor a la determinada para el aislamiento antártico de T. cylindrosporum y para A. album. El TL 50 para las dosis mencionadas fué mayor para los aislamientos evaluados en el presente trabajo que para los resultados de T. cylizdrosporum de Nueva Zelandia.

Soares y Pinnock (1984), evaluaron la infectividad de T. cilindrosporum aislado de Aedes sierrensis en California, USA, contra larvas de esa misma especie. En esos estudios también se demostró la mayor infectividad evidenciada por las blastosporas en relación a los conidios, obteniendo valores de TL 50 con ambos tipos de esporas, inferiores a los que presentaron los aislamientos citados previamente. El TL 50 fue el menor en relación a los aislamientos considerados,con valores bajos para blastosporas, de 5.6 días y 0.6 dfas para conidios y blastosporas respectivamente, con una dosis de $5 \times 10^{5}$ esporos $/ \mathrm{ml}$.

De la comparación de nuestros resultados con los obtenidos por otros autores que utilizaron distintos aislamientos de $T$. cylindrosporum, se deduce que la cepa de suelo antártico y $A$. album, requieren mayor número de días para el desarrollo de la infección, aunque los bajos valores de la DL 50 correspondientes a ambos tipos de esporas y los elevados porcentajes de mortalidad registrados, permiten considerar sus potencialidades como patógenos para el control biológico de mosquitos.

Tabla 1 Mortalidad larval de Culex pipiens con blastosporas y conidios de Aphanocladium album

\begin{tabular}{|c|c|c|c|c|c|c|c|c|c|}
\hline \multirow{2}{*}{$\begin{array}{l}\text { DOSIS } \\
\text { Blast/ml }\end{array}$} & \multicolumn{9}{|c|}{ T I E M P O (DIAS) } \\
\hline & 1 & 5 & 10 & 16 & M.T. & M.C. & DL50 & E.S.DI 50 & TL50 \\
\hline $1 \mathrm{MO} 3$ & 0 & 0 & 60 & 60 & 60 & 58 & & & 10 \\
\hline $1 \mathrm{MO} 4$ & 0 & 0 & 60 & 63 & 63 & 61 & & & 11 \\
\hline $1 \mathrm{MO5}$ & 0 & 50 & 60 & 65 & 65 & 63 & & & 9 \\
\hline $1 \mathrm{MO6}$ & 10 & 50 & 75 & 90 & 90 & 88 & & & 4.38 \\
\hline $1 \mathrm{MO} 7$ & 30 & 70 & 70 & 100 & 100 & 98 & & & 2.25 \\
\hline $1 \mathrm{MO} 8$ & 0 & 50 & 60 & 100 & 100 & 98 & & & 3 \\
\hline Control & 20 & 0 & 0 & 20 & 20 & 0 & $1 \mathrm{MO} 3$ & 0.62 & \\
\hline \multicolumn{10}{|l|}{ Conid./ml } \\
\hline $1 \mathrm{MO} 3$ & 0 & 0 & 15 & 42 & 42 & 40 & & & 12.6 \\
\hline $1 \mathrm{MO} 4$ & 0 & 10 & 28 & 68 & 68 & 66 & & & 10.4 \\
\hline $1 \mathrm{MO} 5$ & 10 & 10 & 25 & 68 & 68 & 66 & & & 10.36 \\
\hline $1 \mathrm{MO6}$ & 0 & 26 & 65 & 86 & 86 & 83 & & & 6.05 \\
\hline 1MO7 & 10 & 56 & 80 & 100 & 100 & 98 & & & 5.9 \\
\hline 1MO8 & 10 & 100 & 100 & 100 & 100 & 98 & & & 2.97 \\
\hline Control & 0 & 20 & $\begin{array}{l}. \quad 20 \\
\therefore\end{array}$ & 20 & 20 & & $1 \mathrm{MO} 3$ & 0.81 & \\
\hline
\end{tabular}

M.T. $=$ Mortalidad larval total $(\%) \quad$ M.C. $=$ Mortalidad larval corregida $\%$ (Formula de Abbott) DL $50=$ Dosis letal 50 E.S. $=$ Error standard T.L.50 $=$ Tiempo letal 50 (días) 
Tabla 2 Mortalidad larval de Culex pipiens con blastosporas y conidios de Tolypocladium cylindrosporum

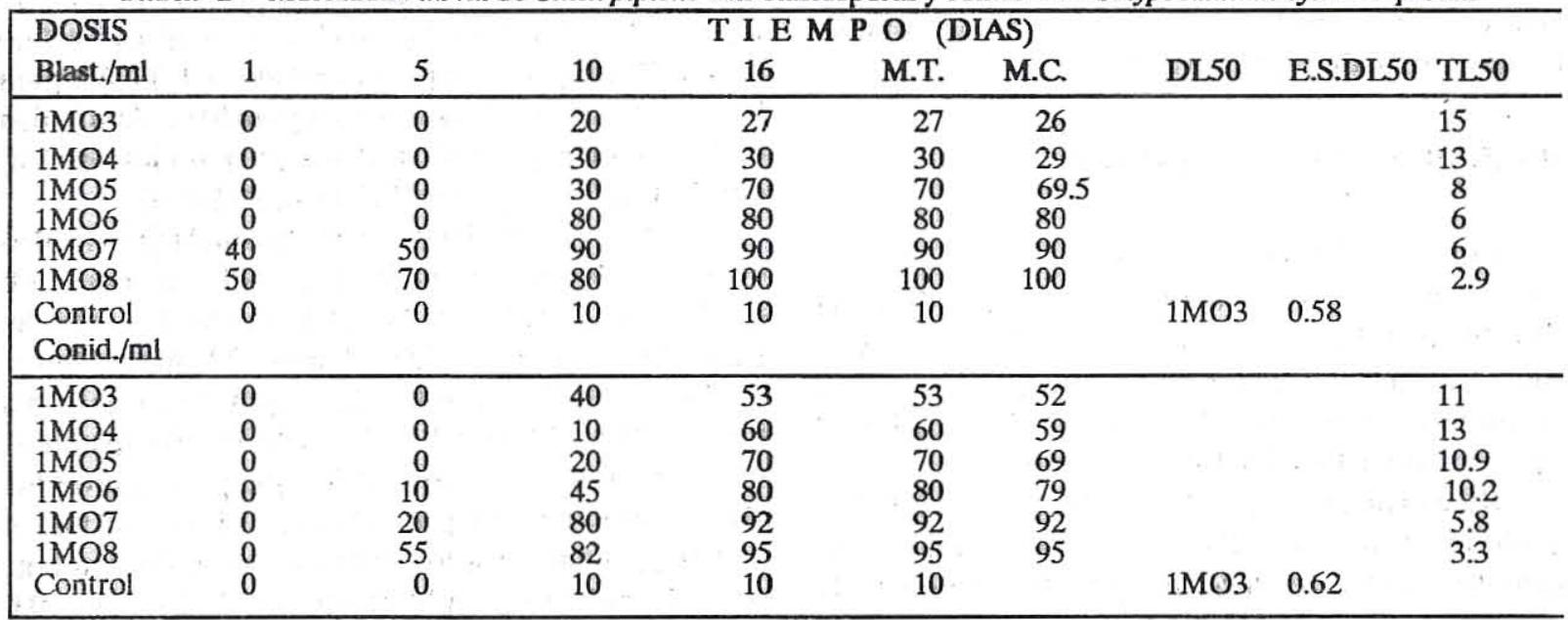

M.T. = Mortalidad larval total (\%) M.C. = Mortalidad larval corregida \% (Formula de Abbott) DL50 = Dosis letal 51 E.S. = Error standard T.L.50 = Tiempo letal 50 (días)

\section{REFERENCIAS}

1.-Abbot, W. S., (1925). A method for computing the effectiveness of an insecticide. J. Econ. En-tomol. 18:265-267.

2.-Biever, K. D. \& Hosteter, D. L. (1971). Activity of the Nuclear Polyhedrosis virus of the Cabbage Looper evaluated at programmed temperature regimen Jour. Inv. Path. 18: 81-84.

4.-Gardner, J.M. \& PIlla!, J.S. (1987) Tolypocladium cylindrasporum (Deuteromycotina. Moniliales) a fungal pathogen of the mosquito Aedes australis III Fields trials against two mosquito species. Mycopathologia 97: 83-88.

5.-Gardner,J.M. \& Ram, R.C., Kumar, S. \& Pillai, J.S. (1986). Field trials of Tolypocladium cylindrosporzm against larvae of Aedes polynesiensis breeding in crab holes in Fiji. J. Am. Mosq. Control. Assoc. 2: 292-295.

6.-Goettel, M.S. (1987 a) Preliminary field trials with the entomopathogenic Hyphomycete Tolypocladium cylindrosporum in Central Alberta. J. Am. Mosq. Contr. Assoc. 3: 239-245.

7.- $\quad$ (1987 b). Studies on biossay of the entomopathogenic Hyphomycete Tolypocladium cylindrosporum in mosquitoes. J. Am. Mosq. Control Assoc. 3 (4): 561-567.

8.-Lopez Lastra, C. C. (1990). Primer registro de Aphanocladium album (Deuteromycotina. Hyphomycetes) como pat6geno de insectos en la República Argentina. Bol. Soc. Arg. Bot. 26 (3-4): 259-261.

9.-Lopez Lastra, C. C. , Reboredo, G. R. \& Spinedi, H. A. (1991). Primer registro de Tolypocladium cylindrosponum Gams (Deuteromycotina. Hyphomycetes) para la Antartida. Consideraciones preliminares sobre su patogenicidad en larvas de Culexpipiens L. (Diptera. Culicidae). Contrib. I.A.A. 39211 p.
10.-Pinnock, D. E., García, R. \& Cubbin, C. M. (1973). Beauveria tenella as a control agent for mosquito larvae.J.Inv Path 22: 143-147.

11.-Reed, L J. \&Muench, H. (1938). A simple method for stimat the end fifty percent point. Am. J. Hyg. 27 (3): 493-497.

12.-Riba, G., Keita, A Soares, Jr. G. G. \& Ferron, P.'(1986). Com. parative studies of Metarhizium anisopliae and Tolipo. cladium cylindrosporum as pathogens of mosquito larvae Jour. The AM. Mosq. Contr. Assoc. 2 (4): 469-473.

13.-Soares, Jr. G. G., Pinnock, D. E. \& Samson, R.A. (1979) Toly. pocladium a new fungal pathogen of mosquito larvae with promise for use in microbial control. Proceedings $47 \mathrm{th}$ Ann. Conf. Mosquit. and Vector Contr. Assoc. January 28-31. 1979.

14.-Soares, Jr. G. G. \& Duddley, E. P. (1984). Effect of temperature on germination, growth and infectivity of the mosquito pathogen Tolypocladium cylindrosporum (Deuteromy. cotina, Hyphomycetes) Jour. of Inv. Path. 43,242-247.

15.-Soares, Jr., G. G. , Rłba, G., Caudal, A. \& Vincent, J.J. (1985). Comparative studies of eleven isolates of the fungal entomopathogen Tolypocladium cylindrosporum and two isolates of T. extinguens. J. Inv. Path. 46: 115-120.

16.-Weisser, J. \& Pillai, J. S. (1981). Tolypocladium cylindrosportam Gams (Deuteromycetes. Moniliales) a new pathogen of mosquito larvae. Entomophaga 26:357-361

17.-Yu, H., Cho, H.W. \& Pillai J.S. (1980). Infection studies of mosquito pathogen Culicinomyces sp. against Aedes and Culex larvae.Korean J. Entomol. 10: 62-63. 